\title{
Building Bridges, Burning Bridges: The Use of Boundary Objects in Agile Distributed ISD Teams
}

\author{
Stephen McCarthy \\ University College Cork \\ Stephen.McCarthy@ucc.ie
}

\author{
Paidi O'Raghallaigh \\ University College Cork \\ P.OReilly@ucc.ie
}

\author{
Ciara Fitzgerald \\ University College Cork \\ CFitzgerald@ucc.ie
}

\author{
Frédéric Adam \\ University College Cork \\ FAdam@ucc.ie
}

\begin{abstract}
Boundary objects are physical and abstract artefacts which support team interactions across diverse knowledge domains. Despite their relevancy, research into the effectiveness of boundary objects in agile distributed ISD remains nascent. In this paper, we develop a framework to theorize their effectiveness in generating cohesion within distributed teams. Our indepth case study finds that their effectiveness is influenced by the nature of the boundary objects themselves but also by team members' willingness to address differences in contextual factors. We identify three contextual factors - structure, identity, and culture - that are critical to supporting team members crossing knowledge boundaries within distributed teams. Furthermore, findings suggest that while boundary objects can indeed foster team cohesion in agile distributed ISD, there are other less explored aspects to their role. For example, we find that boundary objects can be useful for enabling constructive team conflict.
\end{abstract}

\section{Introduction}

Agile distributed information systems development (ISD) is a complex socio-technical practice involving actors from diverse geographical, organizational, and professional backgrounds [1, 2, 3]. Agile distributed ISD seeks to promote emergent and exploratory dialogue between distributed team members to gain a more complete perspective on user requirements, as well as solution features that meet these requirements [4]. Agile distributed ISD teams must therefore continuously interact across disciplinary and geographical boundaries in order to share knowledge, negotiate resources, and resolve uncertainties during rapid iterations of development [2, 3, 4].

To support this dialogical process, ISD team members typically interact using a variety of physical and abstract artefacts, such as project plans, design documentation, and software prototypes [5, 6, 7]. These artefacts can act as boundary objects that facilitate "understanding and cooperation across diverse knowledge domains" by highlighting commonalities, differences, and dependencies in team members' knowledge. [5, pg. 570]. This in turn allows team members to communicate and negotiate knowledge to reach a shared understanding of the proposed system $[8$, 9].

Existing literature has shown how boundary objects can be used to generate cohesion by aligning team members around tasks related to the object of understanding i.e. the proposed system [3, 10, 11, 12]. In particular, studies suggest that boundary objects can promote a 'common lexicon', 'common meaning', and 'common interests' among team members from diverse backgrounds [9]. In addition, boundary objects can offer a common visual representation that mitigates conflict, facilitates decision-making, and resolves disagreements between team members [10]. Similarly, it has been suggested that boundary objects can offer a way of reducing the duration of conflict in virtual teams by mediating conflict identification and resolution [11].

However, existing literature has yet to explore the contextual factors which enable or constrain the effectiveness of boundary objects in agile distributed ISD [3], and how they contribute to team cohesion. This presents opportunities for further research on the effectiveness of boundary objects especially given that agile distributed ISD teams are expected to maintain high levels of interactions despite limited opportunities for face-to-face communication. In this paper, we take steps in this direction by addressing the following research question: What contextual factors enable and constrain the effectiveness of boundary objects in generating cohesion in agile distributed ISD teams?

The remainder of the paper is structured as follows: Section 2 provides the background to the paper and Section 3 outlines our theoretical development. Section 4 introduces the research design behind the in-depth case study and Section 5 presents the findings. Section 
6 offers a discussion of those insights relevant to academia and practice. Section 7 concludes the paper.

\section{Background}

Agile ISD teams seek to develop novel systems using agile methodologies, such as Scrum or Extreme Programming, which emphasize ongoing team interactions, iterations of working software, and close customer collaboration [1, 2, 13]. However, agile distributed ISD teams are characterized by deep social differences in individuals coming from distinct organizational and professional backgrounds [14]. The success of agile distributed ISD therefore depends on team members' ability to share knowledge, negotiate interests, and delegate responsibilities with one another in order to overcome and bridge any knowledge gaps during systems development $[3,4,12,15]$.

Team interactions support cohesion by reconciling the divergent perspectives of distributed team members coming from distinct organizational, geographical, and disciplinary backgrounds $[16,17,18]$. Team cohesion can be defined as the extent to which team members are able to reach a shared understanding of tasks, and their willingness to commit the resources at their disposal, in order to work towards the completion of these required tasks $[17,19,20]$. Team cohesion improves individual performance and also the level of trust between distributed team members, which in turn can promote deeper collaboration [16]. However, some emerging research suggests that in order for cohesion to emerge, team members must engage in dialogue to bridge contextual differences in their positions, interests, and cultural meanings $[21,22,23,24]$. This dialogical process ensures that team members become aligned through increased team cohesion [17, 25].

Existing literature suggests that boundary objects can provide diverse groups with the 'common ground' required for sharing knowledge across professional and organizational boundaries $[8,9,26]$. Physical and abstract artefacts constitute as boundary objects when they enable ISD team members from various backgrounds (i.e. programmers, domain experts, project managers, analysts) to communicate perspectives around the design of a system through ongoing dialogue around the problem-solution coupling [27]. Boundary objects can provide a shared lexicon to structure knowledge, translate identified problems into targeted solutions, and help negotiate interests [9, 27]. However, boundary objects need not impose a shared meaning on team members, and instead they should be 'plastic' enough to support multiple meanings that can evolve during dialogue $[5,7,27]$.

The performance of agile distributed ISD teams can suffer owing to the limited opportunities for face-to-face interactions. Recent work suggests that boundary objects can act as an effective surrogate for personal communication $[3,12,28]$. Artefacts such as prototypes and use cases can provide team members with a common reference to translate domain knowledge into systems requirements during activities such as requirements elicitation and design [5, 6]. However, the use of boundary objects in agile distributed teams is not without its challenges; for instance, elaboration and clarifications are needed to prevent hand-over and follow-up problems due to differences in agile distributed team members' backgrounds [3]. Research on the use of boundary objects in agile distributed ISD is only now emerging $[3,5,6,11]$, and has yet to investigate how contextual factors enable and constrain the effectiveness of boundary objects.

\section{Theoretical Development}

Agile distributed ISD teams are characterized by heightened contextual differences, given the diversity of backgrounds and knowledge of team members [14]. The situation is further complicated by the limited opportunities that distributed ISD team members have for personal communication to resolve these differences [3]. Building on concepts from Parsons [29] and from Carlile [9], our theoretical framework identifies and describes how contextual factors affect the interactions that take place at knowledge boundaries in agile distributed ISD. The framework, called the Boundary Objects in Context (BOC) framework, assists in examining the effectiveness of boundary objects for generating team cohesion (see Table 1).

Building on the work of Parsons [29], the framework identifies three contextual (macro-level) factors structure, identity, and culture - that shape knowledgebased team interactions. Structure deals with the positions, roles, and rules which shape interactions. Structure can exist at numerous levels, such as within a subgroup, a team, or an organization. Identity deals with the interests which motivate courses of action during interactions. For instance, identity can be derived from organizational, professional, or personal interests. Finally, Culture refers to the shared meanings, values, and assumptions which guide team interactions. Literature differentiates between two primary levels of culture: national and organizational [30]. Our theoretical framework focuses on the latter and looks specifically at how culture can emerge from the organizational settings in which a team is embedded. Differences in national cultures, while noteworthy, are outside the scope of the framework.

Building on the work of Carlile [9], the framework looks at three boundaries which team members must cross during knowledge-based interactions: syntactic, 
semantic, and pragmatic. Syntactic boundaries require team members to develop a shared lexicon which provides a common and stable structure for team members to communicate [7]. Teams consisting of individuals from different backgrounds often do not share a common lexicon, making collaboration difficult [9]. Semantic boundaries require team members to address cultural differences in shared meanings. Cultural differences can impede knowledge-based interactions due to confusion or disagreements around interpretation [9]. For instance, distributed teams are often characterized by diverse organizational cultures, which can lead to misunderstandings during communication [14]. Pragmatic boundaries refer to the need for team members to negotiate differences in their unique interests around the use of knowledge [5, 9]. In particular, individuals from different backgrounds often have vested interests in re-using existing knowledge to solve problems [9].
Figure 1 depicts our conceptual diagram, which elucidates how the three contextual factors and three boundaries relate to one other.

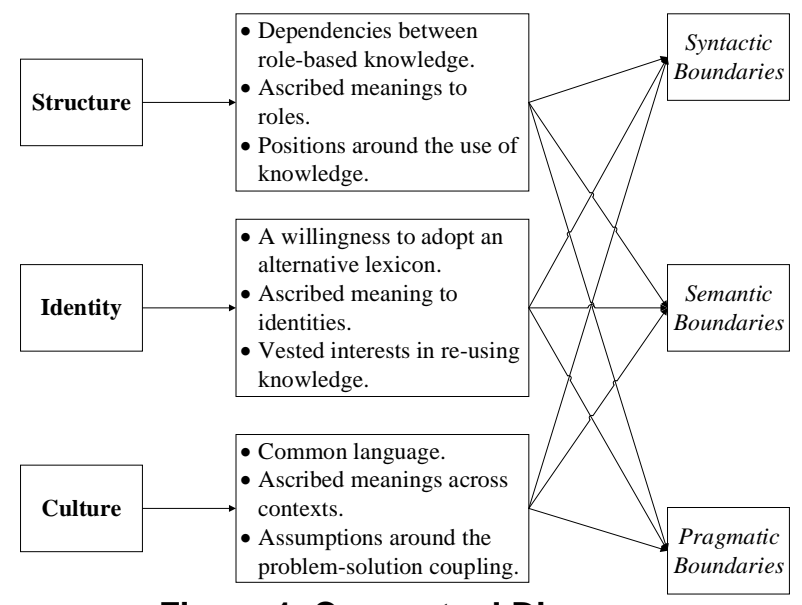

Figure 1. Conceptual Diagram

Table 1: The BOC Framework

\begin{tabular}{|c|c|c|c|}
\hline & Syntactic & Semantic & matic \\
\hline & $\begin{array}{l}\text { Syntactic boundaries require team } \\
\text { members to develop a shared } \\
\text { lexicon, which provides a stable } \\
\text { basis for individuals to } \\
\text { communicate during interactions } \\
{[7,9,27] \text {. Structure shapes how }} \\
\text { team members interact across } \\
\text { syntactic boundaries by creating } \\
\text { dependencies in knowledge. }\end{array}$ & $\begin{array}{l}\text { Semantic boundaries require team } \\
\text { members to resolve differences in } \\
\text { the meanings ascribed to their } \\
\text { positions in order to facilitate } \\
\text { knowledge-based interactions [9]. } \\
\text { Structure enables individuals to } \\
\text { ascribe meaning to their own role } \\
\text { and that of others. }\end{array}$ & $\begin{array}{l}\text { Pragmatic boundaries require } \\
\text { team members to discuss } \\
\text { structural positions in order to } \\
\text { negotiate the use of knowledge } \\
\text { during team interactions. Team } \\
\text { members hold different positions } \\
\text { in their subgroup and wider } \\
\text { organization [22] which create } \\
\text { tensions in individuals' positions. }\end{array}$ \\
\hline & $\begin{array}{l}\text { Knowledge is 'at stake' for team } \\
\text { members from different } \\
\text { backgrounds [9]. Identity shapes } \\
\text { interactions across syntactic } \\
\text { boundaries by influencing whether } \\
\text { or not they are willing to adopt an } \\
\text { alternative lexicon. A shared } \\
\text { syntax is essential to communicate } \\
\text { identities. }\end{array}$ & $\begin{array}{l}\text { Identity shapes semantic } \\
\text { boundaries as individuals ascribe } \\
\text { meaning to their own identity as } \\
\text { well as the identity of others. } \\
\text { Dialogue is needed to highlight } \\
\text { different meanings around the } \\
\text { identity of each individuals and in } \\
\text { order to support knowledge-based } \\
\text { interactions [6]. }\end{array}$ & $\begin{array}{l}\text { Individuals from different } \\
\text { backgrounds have vested interests } \\
\text { in re-using existing knowledge } \\
\text { [6]. To cross pragmatic } \\
\text { boundaries, team members must } \\
\text { negotiate interests around the use } \\
\text { of knowledge [5, 9]. Identity } \\
\text { shapes these boundaries by } \\
\text { fostering diverse interests. }\end{array}$ \\
\hline & $\begin{array}{l}\text { Individuals from the same culture } \\
\text { typically share a common } \\
\text { language; however, interactions } \\
\text { between team members from } \\
\text { different cultures is more difficult } \\
\text { [31]. In order to cross these } \\
\text { syntactic boundaries, team } \\
\text { members must be aware of } \\
\text { language differences. }\end{array}$ & $\begin{array}{l}\text { Semantic boundaries require team } \\
\text { members to address differences in } \\
\text { meanings, which can impede } \\
\text { knowledge-based interactions due } \\
\text { to disagreements in interpretations } \\
{[9,26] \text {. Culture shapes how team }} \\
\text { members generate common } \\
\text { meanings across contexts, creating } \\
\text { divergent values. }\end{array}$ & $\begin{array}{l}\text { Team members must challenge } \\
\text { diverse assumptions around the } \\
\text { problem-solution coupling so as to } \\
\text { cross pragmatic boundaries }[6,9] \text {. } \\
\text { Cultural differences can shape } \\
\text { how team members interact across } \\
\text { pragmatic boundaries and make } \\
\text { negotiation difficult due to } \\
\text { misunderstandings. }\end{array}$ \\
\hline
\end{tabular}

Boundary objects can allow team members to discuss differences in interpretations around the problem-solution coupling and resolve syntactic, semantic, and pragmatic boundaries between individuals' knowledge [6, 9]. For instance, the process of producing or reviewing software prototypes can 
support ongoing dialogue around team members' different lexicons, meanings, and interests through the iterative modification of the IT artefact [5, 9, 27]. This is particularly important in diverse teams, where team members have differing interests around which course of action to pursue [32].

However, boundary objects are not a 'magic bullet'; instead the effectiveness of a boundary object for supporting a common lexicon, meaning, and interest varies across contexts [5]. Therefore, careful consideration ought to be given to the context in which a boundary object is used as well as the ability of individuals to effectively use the boundary object across situations. It is only once a boundary object has been contextualized and meaningfully integrated into localized practices, that it becomes a 'boundary-objectin-use' for various groups [33].

Boundary objects may also be subject to change over time and can adapt to the emerging interpretations of individuals $[5,9]$. Boundary objects can accommodate individuals' unique perspectives as they translate, transfer, and transform knowledge through ongoing interactions [9, 27]. Consequently, boundary objects are evolving rather than static artefacts which are continuously shaped by the contrasting knowledge of individuals. A single boundary object can also adopt different meanings in different contexts and among different individuals; however, they should still retain common features across contexts [9].

\section{Research Design}

An in-depth case study [cf. 34] was undertaken of a six-month agile distributed ISD project which sought to develop a connected health platform for monitoring the wellbeing of expectant mothers across healthcare settings i.e. hospital, GP, and at home. The platform integrated a number of IS artefacts including a smartphone app, certified medical devices for use by expectant mothers, and an Electronic Health Record (EHR) for use mainly by clinicians.

The project team consisted of a multi-disciplinary team of professionals from domains: software engineering, obstetrics, gynecology, and research. The agile distributed ISD project was a collaborative effort consisting of a Principal Investigator (PI), clinical lead, project manager, clinical researcher, research nurse, a full-time and part-time developer, analyst, systems integration engineer, and data architect. In interviews, two subgroups were identified in the team: (1) the 'clinician subgroup', which included the clinical researcher, clinical lead, and research nurse; and (2) 'IT subgroup', which included a project manager, two developers, database architect, and analyst. The clinician and IT subgroups had no prior experience of working together and most team members possessed limited knowledge of connected health.

The team was physically distributed across two cities, and five locations: an innovation center, a local hospital, a large global technology company, a local start-up, and a national health insurer. Team members relied on asynchronous media such as e-mails and JIRA to communicate. The only opportunities for face-to-face interactions were during four workshops organized by the project manager.

Qualitative data was triangulated from participant observations, interviews, and project documents. Between May 2015 and January 2016, the lead author carried out over 700 hours of participatory observations in the field. During this time, four workshops, each lasting six hours, were conducted where the distributed team defined systems requirements. Semi-structured interviews (each lasting about an hour) were conducted with the ten individual team members to gain further insights into the project. The sample included all aforementioned team members who were directly involved in the team, except for the systems integration engineer who was unavailable for interview. Finally, the lead author also had access to project documents, including emails, reports, and project notes, produced throughout the development phase. The unit of analysis was team interactions, while an embedded unit of analysis focuses on the actions and interactions of subgroups within the team.

A directed approach to data analysis was adopted using NVivo 11 to code findings into themes based on the theoretical framework. The lead author identified codes of interest including concepts and properties, as well as their relationship [cf. 35]. The theory building process was guided by the structured case approach which consists of "constructing and articulating a preliminary conceptual structure, collecting and analysing data, and reflecting on the outcomes to build knowledge and theory" [36, pg. 236].

Following the work of Abraham [37], boundary objects were identified as physical project objects possessing the following properties: (i) interpretive flexibility; (ii) identity preservation; (iii) abstraction/concreteness; (iv) stability; (v) modularity based on user-based contextualization; and (vi) visualization. Artefacts may not be intentionally constructed to serve a facilitation purpose, and these properties can emerge to shape interactions [5].

\section{Findings}

It became apparent early in the project that there were some differences in what team members perceived as its primary focus. For instance, the clinicians 
gravitated more towards the clinical research element of the project, while the start-up partner was more interested in the reputational benefits from its involvement in the project. The data architect highlighted these differences: "The end goal was the same for everyone, [but] there were also other goals present in the project. The junior clinician worked on her post doctorate degree but there was no clear sense how this would fit into the project... The [innovation center] had a vision to deliver a research platform however, this did not align with what the [global technology company] wanted". The project manager also recalled his surprise during one meeting when the clinical researcher said that she had not been informed that the IT subgroup would be involved in the project, and "thought she'd be responsible for building the platform", despite having no ISD expertise.

Recognizing the scale of these misunderstandings, the project manager and analyst decided to organize a series of four one-day design workshops to provide team members with an open forum for sharing knowledge and discussing their perspectives on issues. The workshops provided the first opportunity for the team to come together in one physical location for a dedicated length of time to engage in dialogue about the project. The workshops were intended to provide a facilitated, participatory environment for expediting systems requirements gathering. Team members were encouraged to draw on their domain expertise and work together to define the requirements. Three main types of design artifacts - patient journey maps, patient personas, and early prototypes of the proposed EHR - were purposely developed in advance of the workshops to act as a catalyst for expediting the requirements gathering process.

Journey maps depicted healthcare services from the perspective of different personas engaging with the services through a series of 'touch points' shaping the patient experience [38]. Personas refer to fictional caricatures of users, their expectations, prior experiences and anticipated behaviors [39]. Patient journey maps and personas were intended to model the journeys that the personas of expectant mothers would take through a medical pathway illustrating how they would interact with the proposed IT platform and healthcare services. We now look at how boundary objects were shaped by contextual factors (structure, identity, and culture).

\subsubsection{Boundary Objects and Structure}

Use of the journey maps was influenced by team members' positions in the agile distributed ISD team. For instance, prior to the workshops, the IT subgroup had mapped the standard pre-natal appointment schedule for expectant mothers onto a preliminary journey mapping template. This preliminary mapping exercise was guided by the clinical guideline documents for managing hypertensive disorders during pregnancy. However, much to the IT subgroup's surprise, during one workshop the clinical researcher began to use red markers to adjust and cross-out elements on the journey maps. In explaining the changes, the she pointed out that there are always differences in how clinicians in practice implement the proposed clinical guidelines. This interaction allowed the clinician to showcase her medical expertise and assert her importance to the project and her position relative to that of the IT subgroup. This was emphasized by the clinical researcher: "I understood that it was difficult for tech people to envision the patient pathway without the journey map... I didn't always refer to artefacts on the project as the information was more in my mind and I knew the points I wanted to discuss".

It was also clear that the journey maps were used to re-affirm structures within the subgroups. For instance, during one workshop, the clinical lead highlighted to the team the issues with previous drafts of the journey maps that had been produced by the more junior clinical researcher. The clinical researcher had previously agreed requirements for the smartphone app; however, many of these were now overturned by the more senior clinical lead when she attended later meetings. Members of the subgroups noted how she had asserted her position as the senior member of the clinician subgroup. This in turn had a negative impact on the confidence of the clinical researcher who became reluctant to make further decisions without first deferring to the clinical lead.

It also emerged that the journey maps allowed team members, regardless of their positions in the team, an opportunity to challenge decisions. For example, team members were invited by the project manager to challenge how the connected health pathway depicted on the journey maps would improve the existing pathways. This allowed team members, regardless of their position within the team hierarchy, to assert their importance to the project. For instance, the part-time developer, who was in the early stages of his career, utilized the journey maps to challenge the clinical researcher on how usable the proposed smartphone app, blood pressure monitor, and urine analyzer would be for expectant mothers. At another time, the full-time developer took control of editing one of the journey maps put forward by the clinicians to showcase his IT design expertise. He noted how initially "[the clinicians] only saw one (patient) pathway" and his interjection allowed him to propose an alternative and more effective pathway that added new touchpoints 
between the expectant mother and the healthcare system, via the connected health platform.

\subsubsection{Boundary Objects and Identity}

Due to delays in the ethical approval process, the agile distributed team did not have direct access to expectant mothers during early iterations of systems development. The project manager therefore proposed that fictional patient personas would be used to represent the needs of different expectant mothers. Personas included: 'Brenda', who develops white coat hypertension; 'Fiona', who suffers from severe gestational hypertension; and 'Denise', who is hospitalized with severe pre-eclampsia. These personas were used widely during the workshops to help the IT and clinician subgroups to collaborate and build empathy with potential users of the platform. The personas also helped ensure that design discussions centered on patient-centric scenarios. The value of the personas became self-evident when team members started to call out the personas by name when discussing requirements, for instance referring to the name 'Brenda' when speaking about the white-coat hypertension pathway. Personas also helped the team focus on specific instances of patient care rather than a general pathway. However, the developer noted that while personas were useful to the IT subgroup, their value to clinicians was questionable: "... they give us a great understanding of what's going on, like what the system is being used for. It adds a lot of value on the tech side but it didn't [seem to] matter to [the clinical researcher]. It wasn't a blueprint for her as it was already in her head... But it wasn't as clear in her head as on the [design artifacts]". The clinicians believed they were already familiar with the needs of the patients and therefore did not need to engage with the patient personas; however, this assumption was challenged by other team members, who later questioned whether the platform was being driven by the needs of the patients or the needs of clinicians.

Patient personas also created tension between team members' own identities. Patient personas acted as a referee during discussions and allowed team members to engage in constructive conflict around the design of the connected health platform - focusing on what the patient would want, instead of what team members' wanted. In particular, this conflict allowed team members to negotiate the features that expectant mothers would require during the clinical study in light of their personal situations. For instance, the IT subgroup used the patient persona of "Susan", an expectant mother with childcare responsibilities, to role play the personal challenges that she might have taking blood pressure readings at home while attending to young children. The subgroup discussed how the stress of this situation could have a negative impact on Susan's ability to use the platform to take accurate readings. They also discussed the possibility that technophobia could lead to white coat hypertension due to usability difficulties. They role played another scenario involving a persona, Fiona, who visits her elderly parents in the countryside during the weekends. The developer challenged the team to consider the technical challenges that ensue when Fiona takes blood pressure readings but the app is unable to upload her readings as her parents have no Wi-Fi connectivity. These periods of constructive conflict were seen as being important in leading to more creative solutions. They also challenged the personal identities of team members, such as in the case of male developers who role played as technophobic pregnant women. As stated by the project manager: "there's no doubt that personas were incredibly effective... that wasn't by accident; we sat down before each of those workshops trying to figure out how we would break down the barriers between team members, especially between clinical and technical team members and to build empathy for those that were really important - the pregnant women". Personas served to break down some of these barriers.

\subsubsection{Boundary Objects and Culture}

Cultural differences between the IT and clinician subgroups initially made it challenging to agree requirements. As stated by the data architect: "In projects involving different domains you need to learn a new language... I sat down with [the clinical researcher] at one point and asked her to describe elements of the data dictionary... as you need this for the analytics to understand the cause and effect... It's hard for IT staff working in a clinical research space to learn about how things work in the heads of clinicians". The EHR was developed using open source components and plug-ins from open source communities. The forms and charts included in different sections of the EHR in turn stimulated dialogue around what data items were needed, missing, and surplus to requirements. While the EHR had been developed to facilitate dialogue around the 'look and feel' of the system, it also provided an effective means to address cultural differences between subgroups. Cultural differences among the subgroups was particularly evident during communications concerning medical terminology and standardized coding for capturing medical risk factors, symptoms, and pregnancy outcomes on the EHR. The open source solution had adopted the internationally recognized medical vocabularies of ICD-9 and SNO-MED for the EHR. However, it later emerged that these vocabularies did not always conform to the clinicians own localized 
meanings, and in some situations they requested that non-standard items be added to the vocabularies. The EHR prototype emerged as a key design artifact for the negotiation of shared meanings around medical terms. The EHR acted as a 'bridge' between the IT and clinical subgroups, allowing them to translate data items necessary for the clinical study to match the ICD-9 and SNO-MED vocabularies and the local needs of the clinicians.

The EHR also supported constructive conflict between team members. In particular, the EHR was used by the project manager and developer to challenge team members' assumptions around blood pressure and protein thresholds at which alerts would be triggered. The decisions of the clinicians sometimes contradicted the clinical guidelines, which necessitated the team to explore these differences. The EHR was useful for pointing out gaps in the current thinking of clinicians and other team members. For instance, the IT subgroup raised questions around who would be available to react to an alert outside of standard working hours and whether a dedicated staff member would be available to answer 'out of hours' emergency calls from expectant mothers. As stated by one team member: "There's specific challenges in IT where we use back of the envelope calculation of getting a minimum viable solution... [But] in healthcare there's specific challenges because there are lives at stake". In this way the EHR was seen to challenge the cultural assumptions of team members.

\section{Discussion}

Our in-depth findings show how contextual factors such as structure, identity, and culture shapes the effectiveness of boundary objects for achieving team cohesion in agile distributed ISD. Interestingly, we also find the reverse - that the use of boundary objects challenges structures, identities, and cultures. For instance, the 'voices' of personas highlighted differences in identities in the agile distributed team. We suggest that the ability of team members to effectively cross syntactic, semantic, pragmatic boundaries rests on their willingness to use boundary objects to address contextual differences. Table 2 presents a summary some of the main case study findings to highlight examples of where the use of boundary objects interplayed with contextual factors.

Table 2: Summary of Key Findings

\begin{tabular}{|c|c|c|c|}
\hline & Syntactic & Semantic & Pragmatic \\
\hline 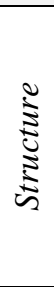 & $\begin{array}{l}\text { Journey maps provided a shared } \\
\text { syntax for team members to reach } \\
\text { agreement around the medical } \\
\text { pathway. Structure impacted use } \\
\text { of the journey maps as team } \\
\text { members asserted their domain } \\
\text { expertise and team role. }\end{array}$ & & $\begin{array}{l}\text { Journey maps allowed team } \\
\text { members to negotiate differences } \\
\text { in knowledge that was 'at stake'. } \\
\text { Conflict between the structure of } \\
\text { subgroups impacted use of the } \\
\text { journey maps as team members } \\
\text { challenged the position of others. }\end{array}$ \\
\hline 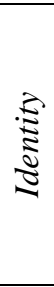 & $\begin{array}{l}\text { The patient personas provided a } \\
\text { shared syntax for understanding } \\
\text { various user groups who were } \\
\text { absent from the ISD process. } \\
\text { Identity shaped the use of personas } \\
\text { as team members discussed } \\
\text { differences in interests. }\end{array}$ & & $\begin{array}{l}\text { Patient personas acted as a referee } \\
\text { during team member discussions } \\
\text { allowing them to address } \\
\text { pragmatic boundaries. Identity } \\
\text { shaped the use of personas as team } \\
\text { members engaged in conflict } \\
\text { around interests. }\end{array}$ \\
\hline$\underset{\mho}{\stackrel{\Xi}{\Xi}}$ & & $\begin{array}{l}\text { The prototype EHR helped cross } \\
\text { semantic boundaries by } \\
\text { supporting shared meanings } \\
\text { around systems requirements. } \\
\text { Differences in culture also shaped } \\
\text { the EHR by transforming the user } \\
\text { interface to match pre-existing } \\
\text { meanings. }\end{array}$ & $\begin{array}{l}\text { The prototype EHR allowed the } \\
\text { team to overcome pragmatic } \\
\text { boundaries around the system } \\
\text { features defined in the project } \\
\text { proposal. Culture shaped the use } \\
\text { of the EHR by creating different } \\
\text { assumptions around the proposal } \\
\text { which needed to be negotiated. }\end{array}$ \\
\hline
\end{tabular}

Agile distributed team members may recognize lexicons, semantics, and interests yet still be unwilling to resolve differences. In the absence of this willingness, 'deep cohesion' remains elusive at best. We define deep cohesion as the situation whereby team members have reached a shared understanding and shared commitment 
to a way forward and to a desired end point. We suggest that this cannot be achieved by resolving syntactic, semantic, and pragmatic boundaries in isolation; instead it requires a multipronged approach which addresses all three, alongside contextual factors. Progress may be impeded by the refusal of certain agile distributed team members to accommodate contextual differences and negotiate lexicons, semantics, and interests. Boundary objects can help address this by offering a means for uncovering contextual differences as well as knowledge boundaries.

Our findings suggest that while boundary objects can offer a useful tool for fostering cohesion between subgroups in agile distributed ISD teams, their role is not limited to this. Similarly, their efficacy does not depend on individuals being in complete agreement. We find that boundary objects can also provide a common point of reference for individuals to challenge one another and to engage in constructive conflict around contextual differences [5]. Existing literature has primarily focused on how boundary objects can be used to promote team cohesion by facilitating common lexicons, meanings, and interests $[9,10,11]$. However, we find that this focus on cohesion overlooks the unique need for constructive conflict in agile distributed ISD teams $[18,21,40]$. We expand on existing literature by contributing new insights into the use of boundary objects in agile distributed ISD.

Team conflict can be defined as the extent to which team members diverge in their shared understanding of and in their utilization of resources for required tasks $[17,41]$. Team conflict challenges the pre-existing assumptions of team members; in particular, moderate levels of conflict can be helpful in allowing individuals to express differences in perspectives, and request clarifications through argumentation [41]. In addition, 'constructive' conflict can help foster creativity by capitalizing on the diversity in knowledge that team members bring with them when completing tasks [42]. This can in turn help team members overcome the knowledge gaps of any one individual and take advantage of the opportunities that divergent knowledge provides for development systems.

We corroborate some emerging research that suggests team conflict is an inherent but also important feature of agile ISD teams $[18,40]$. In our in-depth case study, we find that agile distributed ISD teams are unique in that they face an inevitable need to foster adaptability in changing environments by constantly reframing the problem-solution coupling and embracing divergent perspectives. This is also supported by existing agile ISD scholars who point to the need to clarify assumptions and to prevent the risk of excessive cohesion that impede team members' ability to put forward alternative interests and meanings. For instance, McAvoy and Butler [18] warn against the dangers of excessive levels of cohesion in agile software development projects and they assert the need for a 'devil's advocate' role to breed conflict and to enable the appraisal of alternatives. However, existing literature has not focused on the contextual factors which shape the use of boundary objects for challenging knowledge boundaries.

Interdisciplinary projects require team members to continuously engage in dialogue in order to bring in divergent ideas around constraints, opportunities, and risks associated with the project [31]. As we see in our findings, boundary objects (i.e. journey maps, patient personas, and the EHR) supported periods of both cohesion and conflict that utilized and challenged the interdisciplinary knowledge within the team in order to deliver the connected health platform. For example, privacy regulations affected all areas of the project and therefore the principles of patient confidentiality (clinical) and data protection (technical) needed to be given equal consideration in ISD and the clinical study.

Prior research has described how boundary objects can assume different purposes across different settings [9]. We find supportive evidence that boundary objects can serve different purposes across settings for members of an agile distributed ISD team. We found that boundary objects can serve a novel purpose, different to the one they were originally designed to accommodate. For instance, in the project, it was envisioned that the journey maps would only be used episodically and then discarded once their purpose had been served and team cohesion around users' journeys was reached. However, in interviews it emerged that the journey maps had been used on an ongoing basis as a tool for constructive conflict around the structure of the agile distributed ISD team. In particular, the findings show that the journey maps were employed by team members to challenge others' roles in the agile distributed ISD team and assert their domain expertise relative to others.

However, our research also highlights many challenges associated with the use of boundary objects in agile distributed ISD. For instance, the emergence of team cohesion during the design workshops later proved to be somewhat illusory when a backlog of assigned actions to team members remained outstanding. The emergence of scope creep later in the project also called into question the ability of the boundary objects to generate sustained cohesion around requirements; in particular, the contradictory requirements generated by the clinical researcher and clinical lead pointed to the need for closing out the design specification when everyone is present in the room. Lastly, team members' varying engagement with boundary objects inhibited progress. In contrast to the IT subgroup, once the 
workshops had concluded clinicians did not see the need to refer back to the journey maps, patient personas, and prototype EHR. This became somewhat problematic when the analyst and project manager tried to gain signoff on requirements. It transpired that the clinicians retained no record (other than what was in their heads) of what had been agreed.

\section{Conclusion}

Agile distributed ISD is a complex activity requiring team members to continuously combine individual and organizational knowledge across boundaries [3, 6]. Knowledge-based interactions are important for generating cohesion around the problem-solution coupling. In this paper, we investigated the factors which affect how boundary objects are used to cross knowledge boundaries in agile distributed ISD. In terms of theoretical contributions, we developed a novel theoretical lens, called the BOC framework, to examine how boundary objects are affected by the contextual factors of structure, identity, and culture. These insights are particularly relevant for agile distributed ISD teams where individuals from very diverse backgrounds are expected to continuously interact across boundaries.

In terms of practical contributions, we offer empirical insights into how boundary objects can be used in agile distributed ISD teams to generate requirements and designs based on dialogue between distributed team members. Our findings suggest that boundary objects can support such an approach; yet, we also found that the impact of such boundary objects is often unexpected. For instance, despite their intended purpose, the personas and journey maps ended up becoming a means of both disrupting and maintaining the team structure.

Lastly, contrary to existing literature, our in-depth case study provides new insights into how boundary objects can be used to support periods of both team cohesion and conflict. We suggest that boundary objects can allow team members to address differences in contextual factors through focused periods of team conflict. Team cohesion is not enough, and agile distributed ISD also require team members to challenge underlying assumptions and breed creativity.

One limitation of our study was the nature of the environment in which our in-depth case study took place. The boundary objects were specifically tailored to the healthcare sector by an interdisciplinary team of clinicians and IT professionals. Future research can consider the role of boundary objects for cohesion and conflict in other agile distributed ISD environments. Future research can also look at how the use of boundary objects may gradually change during agile distributed ISD team interactions, and how they may be repurposed in different contexts.

\section{References}

[1] J. S. Persson, L. Mathiassen and I. Aaen, "Agile distributed software development: enacting control through media and context", Information Systems Journal, 22 (2011), pp. 411-433.

[2] B. Ramesh, K. Mohan and L. Cao, "Ambidexterity in agile distributed development: an empirical investigation", Information Systems Research, 23 (2012), pp. 323-339. [3] J. K. Blomkvist, J. Persson and J. Åberg, Communication through boundary objects in distributed agile teams, Proceedings of the 33rd Annual ACM Conference on Human Factors in Computing Systems, ACM, 2015, pp. 1875-1884. [4] S. Sarker and S. Sarker, "Exploring agility in distributed information systems development teams: An interpretive study in an offshoring context", Information Systems Research, 20 (2009), pp. 440-461.

[5] B. Doolin and L. McLeod, "Sociomateriality and boundary objects in information systems development", European Journal of Information Systems, 21 (2012), pp. 570-586.

[6] C. Rosenkranz, H. Vranesic and R. Holten, "Boundary interactions and motors of change in requirements elicitation: A dynamic perspective on knowledge sharing", Journal of the Association for Information Systems, 15 (2014), pp. 306.

[7] A. Weeger, How Do Project-related Artefacts Qualify for Bridging Boundaries in IS Implementation Projects-An Activity Theoretical Perspective, Proceedings of the 50th Hawaii International Conference on System Sciences, 2017. [8] B. A. Bechky, "Sharing meaning across occupational communities: The transformation of understanding on a production floor", Organization science, 14 (2003), pp. 312330.

[9] P. R. Carlile, "Transferring, translating, and transforming: An integrative framework for managing knowledge across boundaries", Organization science, 15 (2004), pp. 555-568. [10] L. J. Black and D. F. Andersen, "Using visual representations as boundary objects to resolve conflict in collaborative model-building approaches", Systems Research and Behavioral Science, 29 (2012), pp. 194-208.

[11] J. Iorio and J. E. Taylor, "Boundary object efficacy: The mediating role of boundary objects on task conflict in global virtual project networks", International Journal of Project Management, 32 (2014), pp. 7-17.

[12] R. Wohlrab, P. Pelliccione, E. Knauss and M. Larsson, "Boundary objects and their use in agile systems engineering", Journal of Software: Evolution and Process, 31 (2019), pp. e2166.

[13] K. Conboy, "Agility from first principles: Reconstructing the concept of agility in information systems development", Information Systems Research, 20 (2009), pp. 329-354.

[14] H. Holmström, B. Fitzgerald, P. J. Ågerfalk and E. Ó. Conchúir, "Agile practices reduce distance in global software 
development", Information systems management, 23 (2006), pp. 7-18.

[15] S. Sawyer, P. J. Guinan and J. Cooprider, "Social interactions of information systems development teams: a performance perspective", Information Systems Journal, 20 (2010), pp. 81-107.

[16] V. Venkatesh and J. B. Windeler, "Hype or help? A longitudinal field study of virtual world use for team collaboration", Journal of the Association for Information Systems, 13 (2012), pp. 735.

[17] X. Yang, Y. Tong and H. H. Teo, "Fostering Fastresponse Spontaneous Virtual Team: Effects of Member Skill Awareness and Shared Governance on Team Cohesion and Outcomes", Journal of the Association for Information Systems, 16 (2015), pp. 919.

[18] J. McAvoy and T. Butler, "The role of project management in ineffective decision making within Agile software development projects", European Journal of Information Systems, 18 (2009), pp. 372-383.

[19] J. B. Windeler, L. M. Maruping, L. P. Robert and C. K. Riemenschneider, "E-profiles, conflict, and shared understanding in distributed teams", Journal of the Association for Information Systems, 16 (2015), pp. 608. [20] S. McCarthy, Exploring the Factors which Affect Cohesion and Conflict in Distributed Information Systems Development Project Teams, University College Cork, 2019. [21] S. McCarthy, P. O'Raghallaigh, C. Fitzgerald and F. Adam, Theorising Antecedents of Cohesion and Conflict in Distributed ISD Project Teams, International Conference on Information Systems, San Francisco, 2018.

[22] S. McCarthy, P. O'Raghallaigh, C. Fitzgerald and F. Adam, Towards a framework for shared understanding and shared commitment in agile distributed ISD project teams, Proceedings of the 27th European Conference on Information Systems, AIS Electronic Library (AISeL), 2019. [23] S. McCarthy, P. O'Raghallaigh, C. Fitzgerald and F. Adam, Distributed ISD Team Leadership and the Paradox of Cohesion and Conflict, Hawaii International Conference on Systems Science, Maui, Hawaii, 2019.

[24] S. McCarthy, P. O'Raghallaigh, C. Fitzgerald and F. Adam, Exploring the Nuances of 'Wickedness' in Information Systems Development, Hawaii International Conference on Systems Science, 2018.

[25] J. Conklin, Dialogue mapping: Building shared understanding of wicked problems, Wiley, West Sussex, UK, 2005.

[26] S. L. Star, "This is not a boundary object: Reflections on the origin of a concept", Science, Technology, \& Human Values, 35 (2010), pp. 601-617.

[27] S. L. Star and J. R. Griesemer, "Institutional ecology, translations' and boundary objects: Amateurs and professionals in Berkeley's Museum of Vertebrate Zoology, 1907-39", Social studies of science, 19 (1989), pp. 387-420. [28] R. Wohlrab, P. Pelliccione, E. Knauss and M. Larsson, Boundary objects in agile practices: Continuous management of systems engineering artifacts in the automotive domain, Proceedings of the 2018 International Conference on Software and System Process, ACM, 2018, pp. 31-40.

[29] T. Parsons, The Social system, Routledge, London, 1951.
[30] D. E. Leidner and T. Kayworth, "A review of culture in information systems research: Toward a theory of information technology culture conflict", MIS quarterly, 30 (2006), pp. 357-399.

[31] M. Strober, Interdisciplinary conversations: Challenging habits of thought, Stanford University Press, 2011.

[32] P. Jarzabkowski and S. Kaplan, "Strategy tools-in-use: A framework for understanding "technologies of rationality" in practice", Strategic Management Journal, 36 (2015), pp. 537-558.

[33] N. Levina and E. Vaast, "The emergence of boundary spanning competence in practice: implications for implementation and use of information systems", MIS quarterly (2005), pp. 335-363.

[34] P. Darke, G. Shanks and M. Broadbent, "Successfully completing case study research: combining rigour, relevance and pragmatism", Information systems journal, 8 (1998), pp. 273-289.

[35] M. B. Miles and A. M. Huberman, Qualitative data analysis: A sourcebook, Sage, Beverly Hills, 1994.

[36] J. M. Carroll and P. A. Swatman, "Structured-case: a methodological framework for building theory in information systems research", European Journal of Information Systems, 9 (2000), pp. 235-242.

[37] R. Abraham, Enterprise architecture artifacts as boundary objects-A framework of properties, Proceedings of the 21st European Conference on Information Systems, Utrecht, Netherlands, 2013.

[38] K. N. Lemon and P. C. Verhoef, "Understanding customer experience throughout the customer journey", Journal of Marketing, 80 (2016), pp. 69-96.

[39] C. LeRouge, J. Ma, S. Sneha and K. Tolle, "User profiles and personas in the design and development of consumer health technologies", International journal of medical informatics, 82 (2013), pp. e251-e268.

[40] T. Dyba and T. Dingsoyr, "What do we know about agile software development?", IEEE software, 26 (2009), pp. 6-9.

[41] P. Van den Bossche, W. Gijselaers, M. Segers, G. Woltjer and P. Kirschner, "Team learning: building shared mental models", Instruct. Science, 39 (2011), pp. 283-301. [42] J.-L. Farh, C. Lee and C. I. Farh, "Task conflict and team creativity: a question of how much and when", Journal of Applied Psychology, 95 (2010), pp. 1173. 